\title{
Onset of Buckling in Drying Droplets of Colloidal Suspensions
}

\section{Citation}

Tsapis, N., E. R. Dufresne, S. S. Sinha, C. S. Riera, J. W. Hutchinson, L. Mahadevan, and D. A. Weitz. 2005. "Onset of Buckling in Drying Droplets of Colloidal Suspensions." Physical Review Letters 94 (1). https://doi.org/10.1103/physrevlett.94.018302.

\section{Permanent link}

http://nrs.harvard.edu/urn-3:HUL.InstRepos:41417296

\section{Terms of Use}

This article was downloaded from Harvard University's DASH repository, and is made available under the terms and conditions applicable to Other Posted Material, as set forth at http:// nrs.harvard.edu/urn-3:HUL.InstRepos:dash.current.terms-of-use\#LAA

\section{Share Your Story}

The Harvard community has made this article openly available.

Please share how this access benefits you. Submit a story.

Accessibility 


\title{
Onset of Buckling in Drying Droplets of Colloidal Suspensions
}

\author{
N. Tsapis, ${ }^{*}$ E. R. Dufresne, ${ }^{\dagger}$ S. S. Sinha, C. S. Riera, J. W. Hutchinson, L. Mahadevan, and D. A. Weitz \\ DEAS and Department of Physics, Harvard University, Cambridge, Massachusetts 02138, USA
}

(Received 2 August 2004; published 3 January 2005)

\begin{abstract}
Minute concentrations of suspended particles can dramatically alter the behavior of a drying droplet. After a period of isotropic shrinkage, similar to droplets of a pure liquid, these droplets suddenly buckle like an elastic shell. While linear elasticity is able to describe the morphology of the buckled droplets, it fails to predict the onset of buckling. Instead, we find that buckling is coincident with a stress-induced fluid to solid transition in a shell of particles at a droplet's surface, occurring when attractive capillary forces overcome stabilizing electrostatic forces between particles.
\end{abstract}

DOI: 10.1103/PhysRevLett.94.018302

PACS numbers: 82.70.Dd, 46.32.+x, 47.55.Dz, 83.50.-v

Drying suspensions show a stunning blend of fluid and elastic behavior. For example, even tiny concentrations of suspended particles can dramatically alter the behavior of a drying droplet. Rather than simply shrinking isotropically, drying colloidal droplets can undergo striking mechanical instabilities, such as fracture and buckling [1]. The basic physics of these instabilities are relatively well understood for elastic materials. However, drying suspensions can be viscoelastic: depending on the experimental time scale, they can either store energy in elastic deformation or relieve stress through viscous flow. In addition, the kinetics of drying drives suspensions far from equilibrium, leading to dramatic changes in material properties as solvent evaporates [2]. Thus, drying droplets pose a number of intriguing fundamental questions. However, they also play an important technological role. For example, spray drying, where fine powders are produced by the rapid drying of aerosols, has become the method of choice for manufacturing large quantities of many foodstuffs, pharmaceuticals, polymers, and detergents $[3,4]$. Despite these broad industrial applications, little is known about the mechanics of drying droplets. Recent experiments investigating drying sessile droplets have shed some light on the problem $[1,2]$, suggesting that the morphologies of buckled colloidal droplets can be interpreted using the theory of thin elastic shells. However, a clear picture of the subtle interplay between forces, structure, and kinetics inside colloidal droplets far from equilibrium has not emerged to describe how drying transforms fluid droplets into buckled elastic shells. Therefore, a new framework is required to understand the mechanical instabilities of drying droplets.

In this Letter, we describe the drying of freely suspended droplets of colloidal suspensions. Initially, these droplets behave like pure liquids and shrink isotropically. Eventually, the droplets buckle like elastic shells. While linear elasticity is able to describe the morphology of buckled droplets, the classic elastic buckling criterion cannot predict the onset of this instability. We present a new framework for understanding buckling in drying colloidal droplets. As droplets dry, a viscoelastic shell of densely packed particles forms at its surface. Initially, the shell yields and thickens as the droplet shrinks. Eventually, the capillary forces that drive deformation of the shell overcome the electrostatic forces stabilizing the particles. At this point, the shell undergoes a sol-gel transition, becomes elastic, and buckles.

We observe the dynamics of drying droplets of aqueous suspensions of monodisperse carboxylate-modified polystyrene colloids (Interfacial Dynamics Corp.). We use the Leidenfrost effect to keep the droplets freely suspended. This effect is familiar to anyone who has sprinkled water on a hot griddle to check the temperature: fluid droplets do not wet surfaces above about $150{ }^{\circ} \mathrm{C}$; rather, they float on a thin layer of their own vapor [5]. The shape of a Leidenfrost droplet is determined by a balance of capillary and gravitational forces. If the droplet radius, $R$, is smaller than the capillary length, $l=\sqrt{\gamma / \rho g}$, surface tension overcomes gravity and the droplet remains spherical [6]. Here, $\rho$ is the density, $g$ is the acceleration due to gravity, and $\gamma$ is the surface tension. For water near $100^{\circ} \mathrm{C}$, the capillary length $l \approx 2.5 \mathrm{~mm}$. Thus, to ensure that they are spherical, we study droplets with radii from 0.8 to $2.2 \mathrm{~mm}$. Using high-speed video, we image the drying of droplets suspended above a concave stainless steel surface maintained at $200^{\circ} \mathrm{C}$. Initially, the colloidal particles have no apparent effect and the droplet shrinks isotropically. Ultimately, the droplets undergo a sudden buckling transition, as shown in Fig. 1(a). The shapes of these buckled droplets closely resemble deflated elastic shells. The observed morphologies are consistent with those obtained from simulations of buckling elastic shells [7] using Surface Evolver [8] [Fig. 1(b)]. Indeed, scanning electron microscopy (SEM) images of fully dried droplets reveal a hollow core surrounded by a buckled shell of densely packed particles, as shown in Fig. 2.

This shell forms as particles pile up just inside the drying droplet's receding air-water interface. The boundary between the shell and the bulk of the droplet is sharp when the time for the fluid to evaporate, $\tau_{\mathrm{dry}}$, is much less than the time required for homogenizing the droplet, $\tau_{\text {mix }}$. The 


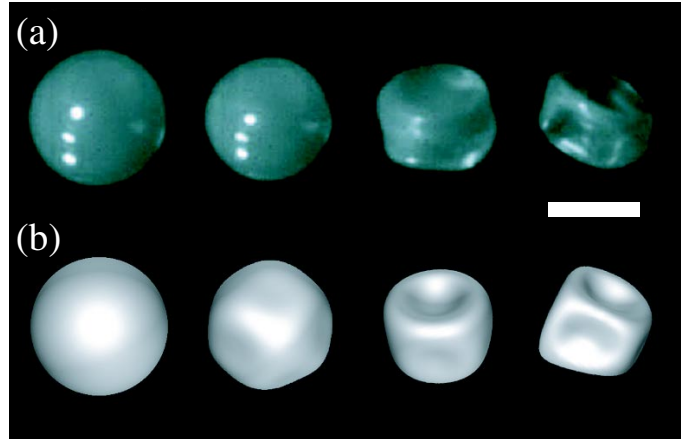

FIG. 1 (color online). Buckling instability in a drying droplet. (a) A colloidal droplet $\left(\phi_{i}=0.4 \%, a=85 \mathrm{~nm}\right.$, and $R_{i}=$ $1.3 \mathrm{~mm}$ ) levitates above a hot plate maintained at $200^{\circ} \mathrm{C}$. Adjacent snapshots are separated by $1.5 \mathrm{~s}$. The second snapshot shows the onset of buckling. The scale bar represents $0.5 \mathrm{~mm}$. (b) Simulation of a buckling elastic shell with $(T / R)_{B}=0.09$ and Poisson ratio 0.4 shows similar morphologies.

mixing time can be estimated as the time a suspended particle takes to diffuse across the radius of the droplet, $\tau_{\text {mix }} \approx R^{2} / D$, where $D$ is the single particle diffusion coefficient. In our experiments, $\tau_{\text {dry }} \approx 60 \mathrm{~s}$ while $\tau_{\text {mix }} \gtrsim$ $10^{3} \mathrm{~s}$. Therefore, the inner core of a droplet remains at its initial volume fraction, $\phi_{i}$, while a shell of thickness $T$ forms with volume fraction $\phi_{c}$. We apply mass conservation to determine the relative thickness of the shell during isotropic shrinkage,

$$
\frac{T}{R}=1-\left[\frac{\phi_{c}-\phi_{i}\left(R_{i} / R\right)^{3}}{\phi_{c}-\phi_{i}}\right]^{1 / 3},
$$

where $R_{i}$ is the initial droplet radius. As the volume of the droplet decreases, new particles join the shell. In addition, the surface area of the droplet shrinks as it dries, forcing the shell to thicken and its particles to rearrange.

Capillary forces drive the deformation of the shell. As water evaporates, tiny menisci form in the gaps between particles at the surface of the shell. The pressure just inside the menisci is $2 \gamma / r_{M}$ below atmospheric pressure, where $r_{M}$ is the local radius of curvature. The tension created by this interfacial curvature simultaneously pulls fluid toward the outer surface and drives particles inward. The radius of curvature, and thus the forces, adjusts to follow fluid evaporation and keep the particles wet.

The shell's response to capillary forces is viscoelastic. During isotropic shrinkage, the viscous nature of the shell dominates as it yields and thickens. However, in order to buckle, the shell must become elastic. Therefore, the onset of buckling must correspond to a crossover from the viscous to elastic regimes of the shell's rheology.

To clarify the relationship between buckling and shell geometry, we explore the variation of the onset of buckling with the initial droplet radius and initial particle volume fraction. These two parameters control the relative thickness of the shell [Eq. (1)]. We identify the onset of buckling

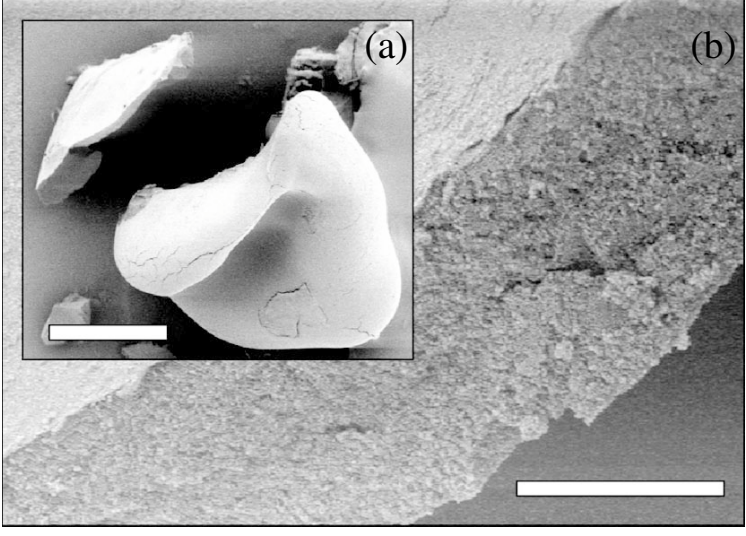

FIG. 2. SEM images of a buckled, dried, and fractured shell created from a suspension of $85 \mathrm{~nm}$ spheres. (a) Scale bar represents $200 \mu \mathrm{m}$. (b) Scale bar represents $10 \mu \mathrm{m}$.

as the instant when the horizontal and vertical radii of the droplet become measurably different. The droplet's radius at the onset of buckling, $R_{B}$, increases linearly with the initial radius of the droplet. Therefore, $R_{B} / R_{i}$ is independent of $R_{i}$, as shown in the inset to Fig. 3(a). The buckling radius scales with the initial particle volume fraction as $\phi_{i}^{1 / 3}$, as shown in Fig. 3(a). The onset of buckling is observable down to remarkably low volume fractions, $\phi \approx$ $10^{-4}$. Applying Eq. (1) to our data, we calculate the relative thickness of the shell at the point of buckling for each initial volume fraction. Notably, we find that $(T / R)_{B}$ is constant over two decades of $\phi_{i}$, as shown in Fig. 3(b). Therefore, the onset of buckling occurs at a critical relative thickness, independent of $R_{i}$ and $\phi_{i}$. Assuming a volume fraction of particles inside the shell equal to 0.58 , we find $(T / R)_{B}=0.09 \pm 0.03$ for particle radii of $a=85 \mathrm{~nm}$. The precise value of $\phi_{c}$ does not affect the scaling of $(T / R)_{B}$ with $\phi_{i}$, but it does affect the absolute values of $(T / R)_{B}$. The inferred value of $(T / R)_{B}$ is consistent with the elastic shell simulations, as shown in Fig. 1(b). For $a=$ $1000 \mathrm{~nm}$, we find $(T / R)_{B}=0.28 \pm 0.14$ and observe slightly different buckling morphologies, as expected for thicker shells.

The thickness of a shell is a key parameter in determining the stress needed to drive its deformation. We relate stress and shell thickness by considering the relative flow of fluid and particles as the droplet shrinks. Modeling the shell as a porous medium, we use Darcy's law to estimate the pressure drop required to drive fluid flow through the particle shell, $\Delta P=\eta T J / k$. Here $\eta$ is the fluid viscosity, $J=d R / d t$ is the volumetric flux of fluid through the shell per unit area, and $k$ is the shell permeability. We estimate the permeability with the Carmen-Kozeny relation $k=$ $\frac{1}{45} \frac{\left(1-\phi_{c}\right)^{3}}{\phi_{c}^{2}} a^{2}$. Combining this picture with Eq. (1) and measurements of $R$ and $d R / d t$ at the point of buckling, we estimate the pressure drop across the shell at the point of buckling, $(\Delta P)_{B}$. Remarkably, the pressure drop is inde- 

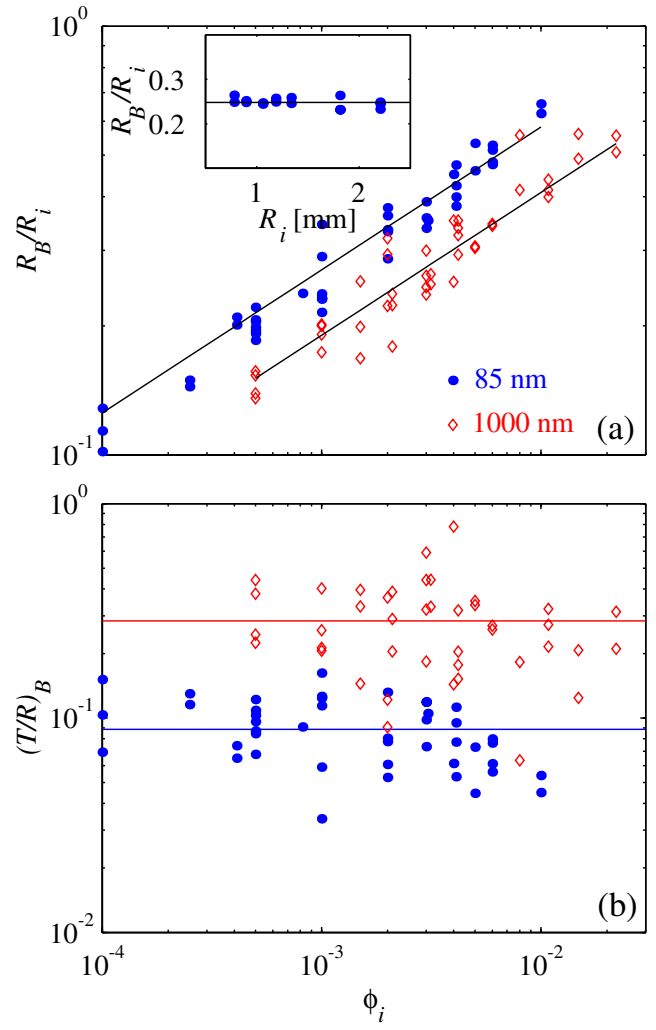

FIG. 3 (color online). Effect of shell geometry on buckling. (a) The radius of the shell at the point of buckling for different initial volume fractions, for spheres of radius 85 and $1000 \mathrm{~nm}$. $R_{i}=1.3 \mathrm{~mm}$. The solid lines show $\phi_{i}^{1 / 3}$ scaling. Inset: the ratio of the buckling radius to the initial radius, $R_{B} / R_{i}$, for a range of initial droplet radii. Particle radius, $a=85 \mathrm{~nm}$. $\phi_{i}=0.002$. (b) The inferred relative thickness of the shell at the point of buckling, $(T / R)_{B}$, for a range of different initial volume fractions.

pendent of the initial volume fraction, as shown in Fig. 4. We find that $(\Delta P)_{B}$ is $(5.0 \pm 1.4) \mathrm{kPa}$ and $(116 \pm 52) \mathrm{Pa}$ for the $85 \mathrm{~nm}$ and $1000 \mathrm{~nm}$ suspensions, respectively.

The emergence of a critical pressure suggests that the crossover from viscous to elastic behavior is initiated by stress. The origin of the critical buckling pressure is clarified by considering the forces acting on individual colloidal particles. Particles confined to the air-water interface are driven together by capillary forces. During isotropic shrinkage, the magnitude of the capillary forces is about $\pi a^{2} \Delta P$, since the pressure drop across the air-water interface is equal to the pressure drop across the shell. Therefore, the critical buckling pressure, $(\Delta P)_{B}$, corresponds to a critical force, $F_{B} \approx \pi a^{2}(\Delta P)_{B}$. We find that $F_{B}=(37 \pm 10) \mathrm{pN}$ and $(120 \pm 50) \mathrm{pN}$ for the small and large spheres, respectively. These forces are of the same order of magnitude as those expected to provide electrostatic stabilization between colloidal particles and can be estimated with measured values of the Hamacker constant and surface potential. The force between two highly

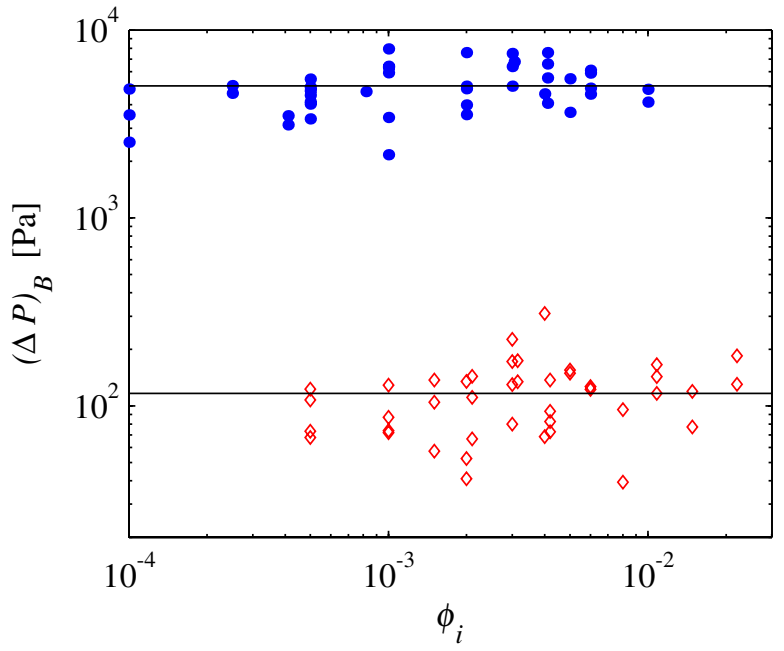

FIG. 4 (color online). Calculated pressure drop at buckling for $85 \mathrm{~nm}$ and $1 \mu \mathrm{m}$ particles, filled and open symbols, respectively.

charged spheres whose surfaces are separated by a distance $D \ll a$ can be estimated as [9]

$$
F(D)=\frac{64 \pi a n_{o} \Gamma^{2} k_{B} T}{\kappa} e^{-\kappa D}-\frac{a A}{12 D^{2}},
$$

where $n_{o}$ is the number density of monovalent electrolyte, $\kappa$ is the Debye screening length, and $A$ is the Hamacker constant. The parameter, $\Gamma=\tanh \left(e \zeta / 4 k_{B} T\right)$, captures the saturation of the electrostatic repulsion with large values of the surface potential, $\zeta$. A combination of electrokinetic and aggregation experiments on similar spheres suggests that $\zeta \approx-100 \mathrm{mV}$ and $A=1.3 \times 10^{-20} \mathrm{~J}$ [10]. Assuming an electrolyte concentration of $1.6 \mu \mathrm{M}$, corresponding to dissolved carbon dioxide, we calculate maximum repulsive forces of about $10 \mathrm{pN}$ and $100 \mathrm{pN}$ for the $85 \mathrm{~nm}$ and $1000 \mathrm{~nm}$ spheres, respectively. This estimate not only captures the magnitude of measured values of $F_{B}$ but also reproduces the observed dependence on particle size.

Our analysis suggests that the forces between particles in a drying droplet play an important role in determining the onset of buckling. We hypothesize that buckling occurs when the capillary forces driving the deformation and flow of a shell overcome the electrostatic forces stabilizing the particles against aggregation. Therefore, at a critical value of the capillary forces, the particles undergo a sol-gel transition, transforming the shell from a viscous fluid to an elastic solid, and triggering the onset of buckling.

We test this picture by modifying the interactions of particles in the shell. We vary the concentration of divalent salt and observe the buckling of a suspension of the $85 \mathrm{~nm}$ particles at an initial volume fraction of $0.4 \%$. As shown in Fig. 5(a), small quantities of divalent salt have a dramatic effect on the morphology of a drying colloidal droplet. Generally, the shells have a more crumpled appearance. This occurs because the shells are thinner, having buckled 

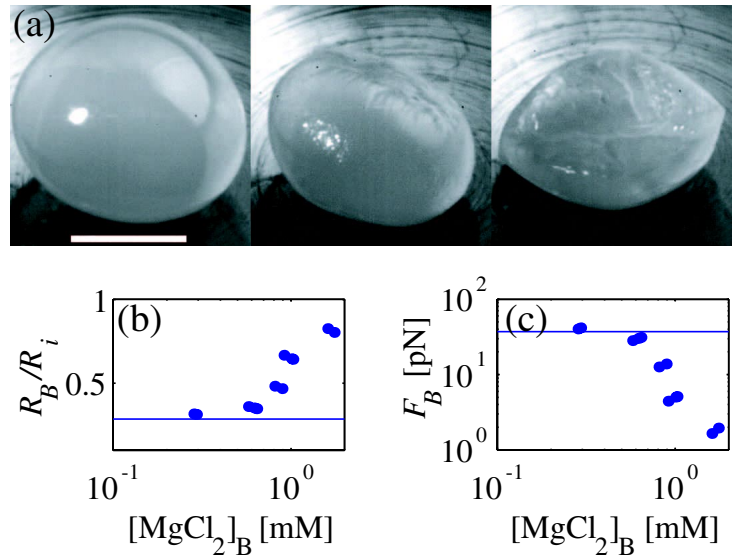

FIG. 5 (color online). Salt-induced buckling. (a) Buckling of a colloidal droplet $\left(\phi_{i}=0.4 \%, a=85 \mathrm{~nm}\right.$, and $\left.R_{i}=1.3 \mathrm{~mm}\right)$ with $1 \mathrm{mM} \mathrm{MgCl} 2$. Adjacent snapshots are separated by $8 \mathrm{~s}$. The scale bar represents $1 \mathrm{~mm}$. (b) The measured buckling radius increases with the concentration of $\mathrm{MgCl}_{2}$. Concentrations of $\mathrm{MgCl}_{2}$ are plotted at the point of buckling, assuming salt remains well mixed during drying. The solid line indicates the buckling radius at no added salt. (c) Calculated interfacial force at buckling with salt concentration. The solid line indicates force at no added salt.

at larger values of $R$, as shown in Fig. 5(b). Calculating the force between particles at buckling, we find that the inferred destabilization force approaches zero as the concentration of $\mathrm{MgCl}_{2}$ approaches $1 \mathrm{mM}$ [Fig. 5(c)]. This can be understood by considering the electrostatic interaction of the magnesium ions with the surface of the spheres. Using Boltzmann statistics, we can estimate the concentration of magnesium ions at the particle surface: $\left[\mathrm{Mg}^{2+}\right]_{\text {surface }}=$ $\left[\mathrm{Mg}^{2+}\right]_{\text {bulk }} \exp \left(2 e \zeta / k_{B} T\right)$. Therefore, as the bulk concentration of $\mathrm{MgCl}_{2}$ approaches $1 \mathrm{mM}$, the concentration of magnesium at the particle surface reaches the solubility limit of about $5 \mathrm{M}$ and condenses to neutralize the particle charge.

This stress-induced fluid to solid transition should be a generic feature of drying suspensions of particles whose interactions have strong short range attractions and relatively long range repulsive stabilizing forces, typical of most aqueous suspensions. By tuning the interactions between particles, the onset of buckling and the morphology of the final shell can be adjusted. This new understanding could provide guidance for engineering the morphology of spray-dried particles. Furthermore, stress-induced gelation may also play an important role in the onset of other elastic instabilities in other geometries, such as the fracture of drying films [11]. Finally, stress-induced gelation may provide a simple means of measuring the forces stabilizing particles in suspension.

We thank J. Bico, M. Brenner, A.-L. Biance, E. Lauga, H. Stone, and D. Quéré for fruitful discussions. We thank the Harvard MRSEC and the NSF for funding through DMR-0213805 and DMR-0243715.

*Present address: UMR CNRS 8612, Centre d'Etudes Pharmaceutiques, Châtenay-Malabry, France.

${ }^{\dagger}$ Corresponding author.

Present address: Dept. of Mechanical Engineering, Yale University, New Haven, CT.

Electronic address: eric.dufresne@yale.edu

[1] L. Pauchard and Y. Couder, Europhys. Lett. 66, 667 (2004).

[2] L. Pauchard and C. Allain, Europhys. Lett. 62, 897 (2003).

[3] K. Masters, Spray Drying in Practice (SprayDryConsult International ApS, Charlottenlund, Denmark, 2002).

[4] N. Tsapis, D. Bennett, B. Jackson, D. A. Weitz, and D. A. Edwards, Proc. Natl. Acad. Sci. U.S.A. 72, 12001 (2002).

[5] J. G. Leidenfrost, De Aquae Communis Nonnullis Qualitatibus Tractatus (Johann Straube, Duisburg, Germany, 1756).

[6] A. L. Biance, C. Clanet, and D. Quéré, Phys. Fluids 15, 1632 (2003).

[7] In these simulations, a droplet's volume is decreased continuously from its initial value in the quasistatic limit so that the shell remains in mechanical equilibrium. At each volume, we determine the shell's shape by minimizing the elastic strain energy using the Cauchy-Green strain matrix and gradient descent method. The shape of the buckled shell is independent of the absolute value of the elastic modulus and depends only on the relative thickness, $T / R$, which determines the relative cost of bending and stretching.

[8] K. Brakke, Exp. Math. 1, 141 (1992).

[9] J. N. Israelachvili, Intermolecular and Surface Forces (Academic Press, New York, 1992).

[10] S.H. Behrens, D. I. Christl, R. Emmerzael, P. Schurtenberger, and M. Borkovec, Langmuir 16, 2566 (2000).

[11] E. R. Dufresne, E. I. Corwin, N.A. Greenblatt, J. Ashmore, D. Y. Wang, A. D. Dinsmore, J.X. Cheng, X.S. Xie, J.W. Hutchinson, and D. A. Weitz, Phys. Rev. Lett. 91, 224501 (2003). 